\title{
Association of hypoalbuminemia with short-term and long-term mortality in patients undergoing continuous renal replacement therapy
}

\author{
Jong Joo Moon ${ }^{\mathbb{D}}$, Yaerim Kim ${ }^{\mathbb{(}}$, Dong Ki Kim ${ }^{\mathbb{1}}$, Kwon Wook Joo ${ }^{\mathbb{D}}$, Yon Su Kim ${ }^{\mathbb{1}}$, Seung Seok Han ${ }^{(\mathbb{D}}$ \\ Department of Internal Medicine, Seoul National University College of Medicine, Seoul, Republic of Korea
}

\begin{abstract}
Background: Hypoalbuminemia reflects several pathological conditions, including nutritional deficiencies and chronic inflammation. However, its relationship with short-term and long-term mortality in patients undergoing continuous renal replacement therapy (CRRT) remains unclear. The present study aimed to assess the effect of hypoalbuminemia on mortality in a large cohort of patients undergoing CRRT.

Methods: The study retrospectively reviewed 1,581 patients who underwent CRRT for the treatment of acute kidney injury from 2010 to 2016. The patients were categorized by tertiles of serum albumin levels at CRRT initiation. The odds ratios and hazard ratios for the risk of all-cause mortality were calculated before and after adjustment for multiple covariates.

Results: The mean albumin level was $2.7 \pm 0.6 \mathrm{~g} / \mathrm{dL}$ at CRRT initiation. During a median follow-up period of 14 days (maximum, 4 years), 1,040 patients (65.8\%) died. The risk of overall mortality was higher in the first tertile group than in the third tertile group (hazard ratio, 1.9 [1.63-2.21]). When the mortality rate was stratified by timeframe, the risk was steadily higher in the first tertile group than in the third tertile group (odds ratios: 3.0 [2.34-3.87] for 2-week mortality, 2.7 [2.12-3.52] for 1-month mortality, 2.7 [2.08-3.53] for 6-month mortality, and 2.8 [2.113.62] for 1-year mortality). Additionally, the rates of intensive care unit mortality and in-hospital mortality were higher in the first tertile group than in the third tertile group.

Conclusion: The initial hypoalbuminemia was independently associated with short-term and long-term mortality in patients undergoing CRRT. Thus, the serum albumin level should be monitored during CRRT.
\end{abstract}

Keywords: Acute kidney injury, Continuous renal replacement therapy, Hypoalbuminemia, Mortality

Received August 20, 2019; Revised October 21, 2019;

Accepted November 19, 2019

Edited by Sejoong Kim, Seoul National University, Seoul, Republic of Korea

Correspondence: Seung Seok Han

Department of Internal Medicine, Seoul National University College of Medicine, 103 Daehak-ro, Jongno-gu, Seoul 03080, Republic of Korea. E-mail: hansway8o@gmail.com

Copyright @ 2020 by The Korean Society of Nephrology

(a) This is an open-access article distributed under the terms of the Creative Commons Attribution Non-Commercial License (http://creativecommons. org/licenses/by-nc-nd/4.0/), which permits unrestricted non-commercial use, distribution, and reproduction in any medium, provided the original work is properly cited.

\section{Introduction}

Acute kidney injury (AKI) is known to increase the mortality rate and prolong hospital stay and healthcare cost [1]. AKI is common among hospitalized patients, and its incidence has been continuously increasing for several decades [1-3]. Approximately $40 \%$ to $60 \%$ of patients who are admitted to the intensive care unit develop AKI $[4,5]$ and require renal replacement therapy [5-7]. Continuous renal replacement therapy (CRRT) is a widely used renal replacement modality, particularly when patients have AKI and are unstable, because it can easily control biochemical imbalance associated with AKI $[8,9]$. Con- 
sidering the critical condition of patients with AKI, the accurate prediction of patient prognosis after CRRT has received increasing attention; however, further research is warranted.

Albumin is involved in many physiological responses, such as osmotic pressure maintenance, drug metabolism [10], immune system regulation [11], and antioxidant effects $[10,12]$. Thus, hypoalbuminemia reflects several pathological conditions, such as organ failure, nutritional deficiency, and inflammation [13,14]. Hypoalbuminemia is identified in up to $30 \%$ of hospitalized patients at admission [15], and it is associated with mortality, prolonged hospital stay, and several complications [1618]. The mortality rates of patients who present on an emergency basis with hypoalbuminemia are three times higher than the mortality rates of patients with normal albumin levels, despite adjustment for the effects of comorbidities [16]. Hypoalbuminemia occurs even more frequently in critically ill patients. However, its relationship with short-term and long-term mortality in patients undergoing CRRT remains unclear. Therefore, the present study aimed to assess the effect of hypoalbuminemia on mortality in a large cohort of patients undergoing CRRT.

\section{Methods}

\section{Patient and data collection}

The study design was approved by the Institutional Review Board of Seoul National University Hospital (approval no. H-1812-069-993) and was conducted in accordance with the principles of the Declaration of Helsinki. The study retrospectively reviewed 2,205 patients who underwent CRRT at Seoul National University Hospital from June 2010 to December 2016. Among them, patients who were under 18 years of age $(n=299)$ and those who had previously undergone maintenance dialysis for endstage renal disease $(n=325)$ were excluded. Finally, 1,581 patients were analyzed in the present study. The need to acquire informed consent from the patients was waived.

We collected the following baseline demographic data: age, sex, weight, AKI cause, dialysis dose, hypertension, diabetes mellitus, ischemic heart disease, cerebrovascular disease, peripheral vascular disease, malignancy, and mechanical ventilation use. The AKI causes were classi- fied into the following four categories: septic, postoperative, nephrotoxic, and other causes. If there appeared to be a direct cause related with surgery and the surgery was performed within 24 hours prior to the initiation of CRRT, the cause was classified as postoperative. Multifactorial AKI was classified according to the main cause. The other causes of AKI included hepatorenal syndrome $(n=46)$, other ischemic injury $(n=356)$, sudden cardiac arrest $(\mathrm{n}=76)$, and others $(\mathrm{n}=189)$. Laboratory data included serum creatinine, hemoglobin, sodium, potassium, and albumin levels. The Acute Physiology Assessment and Chronic Health Evaluation II (APACHE II) score was measured at CRRT initiation [19].

\section{Outcomes}

The primary outcome was all-cause mortality after CRRT. This outcome was further stratified according to the timeframe ( 2 weeks, 1 month, 6 months, and 1 year). With regard to short-term mortality, intensive care unit mortality and in-hospital mortality were also evaluated. The information on all-cause mortality was obtained from the National Database of Statistics Korea.

\section{Statistical analysis}

Categorical and continuous variables are expressed as proportion and mean \pm standard deviation when they were normally distributed and as median with interquartile range when they were not normally distributed, respectively. The normality of the distribution was analyzed using the Kolmogorov-Smirnov test. The chi-square test was used to compare categorical variables. Fisher's exact test was used when the chi-square test was not applicable, and Student $t$ test or the Mann-Whitney $U$ test was used for continuous variables with or without a normal distribution. Nonlinear relationships were explored with a restricted cubic spline analysis. The odds ratios (ORs) and confidence intervals for mortality were calculated using logistic regression analysis. Kaplan-Meier survival curves were drawn and compared using the log-rank test. A Cox proportional hazard regression model was applied to calculate the hazard ratios (HRs) of the risk of mortality. The adjusting variables were as follows: age; sex; weight; AKI cause; target dose; mean arterial pressure; hypertension; diabetes mellitus; history of ischemic heart 
disease, cerebrovascular disease, and peripheral vascular disease; malignancy; mechanical ventilation; laboratory findings, such as creatinine, hemoglobin, sodium, and potassium levels; and the APACHE II score. All statistical analyses were performed using IBM SPSS Statistics (version 23.0; IBM Corp., Armonk, NY, USA). A P value $<0.05$ was considered statistically significant.

\section{Results}

\section{Patient characteristics}

The mean patient age was $63.2 \pm 15.2$ years, and $60.5 \%$ of the patients were male. The proportion of patients with sepsis was $38.7 \%$. Patients were divided into three groups according to the tertiles of the serum albumin levels, and the serum albumin levels were $\leq 2.4,2.5$ to 2.9 , and $\geq 3.0$ $\mathrm{g} / \mathrm{dL}$ from the first to the third tertiles. The AKI cause, dialysis dose, malignancy presence, hemoglobin level, potassium level, and APACHE II score differed among the tertiles. The baseline characteristics are presented in Table 1.

\section{Relationship between serum albumin and mortality}

During a median follow-up period of 16 days (range, 13-19 days; maximum, 4 years), 1,040 patients (65.8\%) died. The mortality incidence was 20.4 deaths per 1,000 person-days. When a nonlinear relationship was applied, the risk of mortality increased as the serum albumin level decreased (Fig. 1). After categorizing the patients into tertiles, a significant gap was noted among the survival curves of the tertiles $(P<0.001$ by log-rank test) (Fig. 2). When a Cox regression model was applied, the risk of

Table 1. Baseline patient characteristics

\begin{tabular}{|c|c|c|c|c|c|}
\hline \multirow[b]{2}{*}{ Characteristic } & \multirow{2}{*}{$\begin{array}{c}\text { Total } \\
(\mathrm{n}=1,581)\end{array}$} & \multicolumn{3}{|c|}{ Albumin group } & \multirow[b]{2}{*}{$P$ value } \\
\hline & & $\begin{array}{l}\text { First tertile } \\
(\mathrm{n}=491)\end{array}$ & $\begin{array}{l}\text { Second tertile } \\
\qquad(n=548)\end{array}$ & $\begin{array}{l}\text { Third tertile } \\
(n=542)\end{array}$ & \\
\hline Age (yr) & $63.2 \pm 15.2$ & $63.2 \pm 15.1$ & $63.8 \pm 15.1$ & $62.6 \pm 15.3$ & 0.326 \\
\hline Male sex (\%) & 60.5 & 61.3 & 61.3 & 59.0 & 0.681 \\
\hline Body weight (kg) & $60.5 \pm 13.1$ & $60.9 \pm 14.1$ & $60.7 \pm 12.6$ & $59.6 \pm 12.5$ & 0.135 \\
\hline Acute kidney injury cause (\%) & & & & & $<0.001$ \\
\hline Sepsis & 38.7 & 56.0 & 41.1 & 20.7 & \\
\hline Surgery & 10.4 & 7.3 & 8.6 & 14.9 & \\
\hline Nephrotoxin & 8.7 & 5.3 & 8.9 & 11.6 & \\
\hline Others & 42.2 & 31.4 & 41.4 & 52.8 & \\
\hline Hemodiafiltration dose $(\mathrm{mL} / \mathrm{kg} / \mathrm{hr})$ & $42.4 \pm 14.0$ & $44.5 \pm 15.3^{c}$ & $43.2 \pm 14.5^{c}$ & $39.7 \pm 11.5$ & $<0.001$ \\
\hline Hypertension (\%) & 28.4 & 26.7 & 30.1 & 28.2 & 0.470 \\
\hline Diabetes mellitus (\%) & 29.0 & 24.4 & 29.9 & 32.1 & 0.021 \\
\hline Ischemic heart disease (\%) & 12.5 & 10.6 & 12.0 & 14.6 & 0.143 \\
\hline Cerebrovascular disease (\%) & 12.1 & 10.6 & 12.2 & 13.5 & 0.367 \\
\hline Peripheral vascular disease (\%) & 7.3 & 6.1 & 7.5 & 8.3 & 0.397 \\
\hline Malignancy (\%) & 36.7 & 42.8 & 37.6 & 30.4 & $<0.001$ \\
\hline Mechanical ventilation (\%) & 84.0 & 87.0 & 82.8 & 82.5 & 0.096 \\
\hline \multicolumn{6}{|l|}{ Blood tests } \\
\hline Creatinine (mg/dL) & $2.7 \pm 1.8$ & $2.6 \pm 1.7$ & $2.8 \pm 1.8$ & $2.7 \pm 1.8$ & 0.392 \\
\hline Hemoglobin (g/dL) & $9.8 \pm 2.2$ & $9.3 \pm 2.1^{c}$ & $9.7 \pm 2.0^{c}$ & $10.3 \pm 2.2$ & $<0.001$ \\
\hline Sodium (mmol/L) & $138.6 \pm 7.9$ & $138.8 \pm 8.4$ & $138.8 \pm 7.7$ & $138.1 \pm 7.7$ & 0.269 \\
\hline Potassium (mmol/L) & $4.3 \pm 0.9$ & $4.4 \pm 1.0$ & $4.2 \pm 0.9^{a}$ & $4.3 \pm 0.8$ & 0.009 \\
\hline Albumin (g/dL) & $2.7 \pm 0.6$ & $2.1 \pm 0.3^{c}$ & $2.7 \pm 0.1^{c}$ & $3.4 \pm 0.4$ & $<0.001$ \\
\hline APACHE II score & $30.4 \pm 9.1$ & $37.5 \pm 9.5^{\mathrm{a}}$ & $35.0 \pm 10.1$ & $35.4 \pm 10.9$ & $<0.001$ \\
\hline Follow-up duration (d) & $12(3-32)$ & $5(1-22)^{b}$ & $14(3-36)$ & $16(5-35)$ & 0.048 \\
\hline
\end{tabular}

APACHE, Acute Physiology and Chronic Health Evaluation.

${ }^{\mathrm{a}} P<0.05 ;{ }^{\mathrm{b}} P<0.01 ;{ }^{\mathrm{C}} P<0.001$ (compared with the third tertile group). 


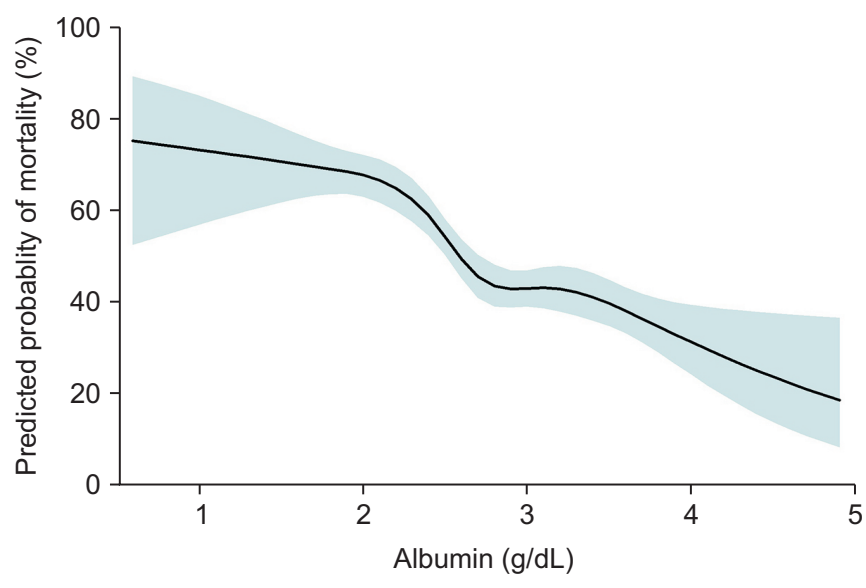

Figure 1. Nonlinear relationship between the serum albumin level and predicted probability of all-cause mortality.

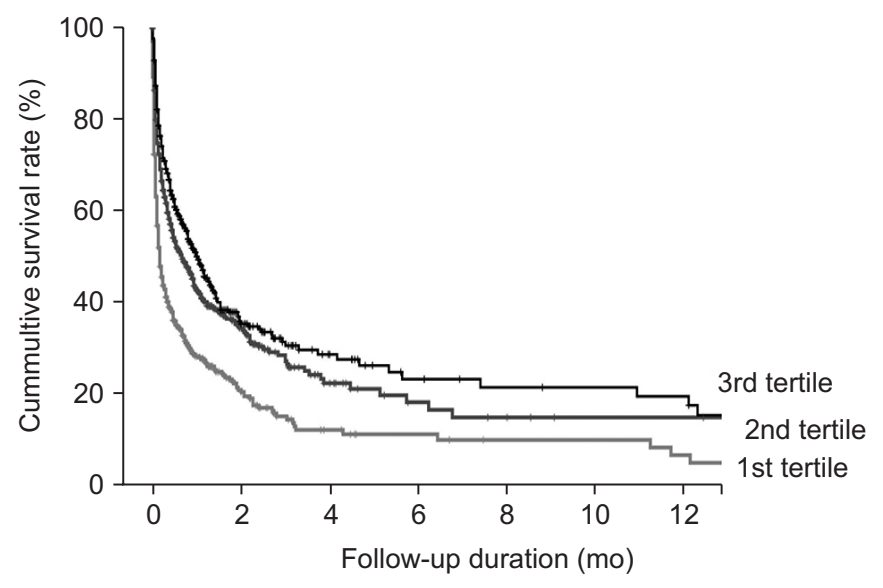

Figure 2. Kaplan-Meier survival curves according to the tertiles of serum albumin.

Table 2. Hazard ratios of all-cause mortality according to the serum albumin levels

\begin{tabular}{|c|c|c|c|c|c|c|}
\hline \multirow{2}{*}{ Albumin group } & \multirow{2}{*}{ Range (g/dL) } & \multirow{2}{*}{ Events (\%) } & \multicolumn{2}{|c|}{ Univariate } & \multicolumn{2}{|c|}{ Multivariate $^{a}$} \\
\hline & & & $\mathrm{HR}(95 \% \mathrm{Cl})$ & $P$ value & HR $(95 \% \mathrm{Cl})$ & $P$ value \\
\hline Third tertile & $\geq 3.0$ & 56.6 & 1 (Reference) & & 1 (Reference) & \\
\hline Second tertile & $2.5-2.9$ & 55.8 & $1.19(1.020-1.386)$ & 0.027 & $1.06(0.904-1.242)$ & 0.472 \\
\hline First tertile & $\leq 2.4$ & 70.7 & $1.90(1.634-2.209)$ & $<0.001$ & $1.57(1.332-1.842)$ & $<0.001$ \\
\hline
\end{tabular}

APACHE, Acute Physiology and Chronic Health Evaluation; $\mathrm{Cl}$, confidence interval; HR, hazard ratio.

${ }^{a}$ Adjusted for age; sex; weight; acute kidney injury cause; target dose; mean arterial pressure; hypertension; diabetes mellitus; history of ischemic heart disease, cerebrovascular disease, and peripheral vascular disease; malignancy; mechanical ventilation; laboratory findings, such as creatinine, hemoglobin, sodium, and potassium levels; and APACHE II score.

mortality was higher in the first tertile group than in the third tertile group, and this remained significant despite adjustment for multiple variables (Table 2).

Although patients who died within 24 hours of CRRT were excluded $(n=247)$, the first tertile group remained at risk of mortality when compared with the finding in the third tertile group (adjusted HR, 1.28 [1.063-1.541]; $P=0.009$ ). When analysis was performed after stratification by the presence of septic AKI, the risk of mortality was higher in the first tertile group than in the third tertile group (adjusted HR, 1.56 [1.223-1.988] for septic AKI; adjusted HR, 1.58 [1.276-1.958] for non-septic AKI).

When the serum albumin level was considered a continuous variable in the multivariate regression model, the risk of mortality showed a 1.7 -fold increase with each $1 \mathrm{~g} / \mathrm{dL}$ decrease in the serum albumin level. When the analysis was stratified according to the timeframes of follow-up, the risk of mortality was higher in the first tertile group than in the third tertile group (ORs: 2.5 [1.224.99] for 24-hour mortality, 2.3 [1.72-3.11] for 1-week mortality, 1.7 [1.40-2.53] for 1-month mortality, and 1.8 [1.31-2.40] for 6-month mortality) (Table 3). When intensive care unit and in-hospital mortalities were evaluated, patients with high albumin levels had a survival benefit when compared with the findings in those with hypoalbuminemia (Table 4 ).

\section{Discussion}

Hypoalbuminemia is associated with increased morbidity and mortality in several pathological conditions. Despite the clinical implications of hypoalbuminemia, its relationship with mortality in patients receiving CRRT has not been demonstrated. To our knowledge, the present study is the first to show that hypoalbuminemia is associated with high mortality after CRRT initiation. This trend was independent of the timeframe of follow-up or the location of death (intensive care unit or hospital ward).

Hypoalbuminemia might result from several pathologi- 
Table 3. Odds ratios ratios of cumulative mortality at different timeframes according to the serum albumin levels

\begin{tabular}{|c|c|c|c|c|c|c|}
\hline \multirow{2}{*}{ Timeframe } & \multirow{2}{*}{ Albumin group } & \multirow{2}{*}{ Events (\%) } & \multicolumn{2}{|c|}{ Univariate } & \multicolumn{2}{|c|}{ Multivariate $^{a}$} \\
\hline & & & OR $(95 \% \mathrm{Cl})$ & $P$ value & OR $(95 \% \mathrm{Cl})$ & $P$ value \\
\hline \multirow[t]{3}{*}{24 hours } & Third tertile & 7.5 & 1 (Reference) & & 1 (Reference) & \\
\hline & Second tertile & 13.5 & $2.07(1.373-3.122)$ & 0.001 & 2.04 (1.015-4.133) & 0.045 \\
\hline & First tertile & 27.4 & $5.03(3.424-7.389)$ & $<0.001$ & $2.46(1.216-4.992)$ & 0.012 \\
\hline \multirow[t]{3}{*}{1 week } & Third tertile & 28.2 & 1 (Reference) & & 1 (Reference) & \\
\hline & Second tertile & 35.2 & $1.38(1.070-1.786)$ & 0.013 & $1.19(0.896-1.580)$ & 0.230 \\
\hline & First tertile & 55.8 & $3.21(2.480-4.156)$ & $<0.001$ & $2.31(1.715-3.105)$ & $<0.001$ \\
\hline \multirow[t]{3}{*}{1 month } & Third tertile & 45.7 & 1 (Reference) & & 1 (Reference) & \\
\hline & Second tertile & 54.3 & $1.46(1.150-1.845)$ & 0.002 & $1.12(0.939-1.603)$ & 0.134 \\
\hline & First tertile & 69.2 & $2.73(2.116-3.515)$ & $<0.001$ & $1.70(1.402-2.532)$ & $<0.001$ \\
\hline \multirow[t]{3}{*}{6 months } & Third tertile & 55.0 & 1 (Reference) & & 1 (Reference) & \\
\hline & Second tertile & 63.1 & $1.44(1.136-1.836)$ & 0.003 & $1.18(0.898-1.537)$ & 0.241 \\
\hline & First tertile & 76.4 & $2.71(2.075-3.532)$ & $<0.001$ & $1.77(1.305-2.401)$ & $<0.001$ \\
\hline
\end{tabular}

APACHE, Acute Physiology and Chronic Health Evaluation; $\mathrm{Cl}$, confidence interval; OR, odds ratio.

${ }^{a}$ Adjusted for age; sex; weight; acute kidney injury cause; target dose; mean arterial pressure; hypertension; diabetes mellitus; history of ischemic heart disease, cerebrovascular disease, and peripheral vascular disease; malignancy; mechanical ventilation; laboratory findings, such as creatinine, hemoglobin, sodium, and potassium levels; and APACHE II score.

Table 4. Odds ratios of intensive care unit mortality and in-hospital mortality according to the serum albumin levels

\begin{tabular}{|c|c|c|c|c|c|}
\hline & \multirow{2}{*}{ Albumin group } & \multicolumn{2}{|c|}{ Univariate } & \multicolumn{2}{|c|}{ Multivariate $^{\mathrm{a}}$} \\
\hline & & $\mathrm{OR}(95 \% \mathrm{Cl})$ & $P$ value & OR $(95 \% \mathrm{Cl})$ & $P$ value \\
\hline \multirow[t]{3}{*}{ ICU mortality } & Third tertile & 1 (Reference) & & 1 (Reference) & \\
\hline & Second tertile & $1.29(1.019-1.641)$ & 0.035 & $1.11(0.846-1.442)$ & 0.464 \\
\hline & First tertile & $2.51(1.942-3.251)$ & $<0.001$ & $1.77(1.315-2.374)$ & $<0.001$ \\
\hline \multirow[t]{3}{*}{ In-hospital mortality } & Third tertile & 1 (Reference) & & 1 (Reference) & \\
\hline & Second tertile & $1.37(1.077-1.750)$ & 0.011 & $1.13(0.858-1.479)$ & 0.390 \\
\hline & First tertile & $2.67(2.037-3.498)$ & $<0.001$ & 1.77 (1.299-2.413) & $<0.001$ \\
\hline
\end{tabular}

APACHE, Acute Physiology and Chronic Health Evaluation; Cl, confidence interval; ICU, intensive care unit; OR, odds ratio.

${ }^{a}$ Adjusted for age; sex; weight; acute kidney injury cause; target dose; mean arterial pressure; hypertension; diabetes mellitus; history of ischemic heart disease, cerebrovascular disease, and peripheral vascular disease; malignancy; mechanical ventilation; laboratory findings, such as creatinine, hemoglobin, sodium, and potassium levels; and APACHE II score.

cal conditions, such as malnutrition and inflammation [20]. These conditions might be associated with impaired immunity, the recurrence of hospital-acquired infection [21], or an increase in infection-related death [22]. A hypoalbuminemia-induced inflammatory state has been shown to interfere with proper homeostatic responses in the postoperative period [20] and affect surgical outcomes $[23,24]$. The patients with hypoalbuminemia in the present study might have had inflammatory or impaired immune milieu before CRRT, with subsequent high mortality.

Albumin has an antioxidant property that defends against oxidative stress [12]. After ischemic stroke, patients with hypoalbuminemia display a poor prognosis because of impaired neuroprotection against oxidative stress [25]. In patients with kidney disease, excessive oxidative stress can induce the development and progression of injury $[26,27]$. Thus, patients with hypoalbuminemia are at risk of AKI and chronic kidney disease $[18,28]$. According to these mechanisms, the patients with hypoalbuminemia in the present study might not recover organ function in the long-term, including kidney function, and might have a high risk of mortality.

As hypoalbuminemia has a negative effect on patient prognosis, the use of albumin replacement therapy has been continuously increasing for many years [29]. The first randomized controlled trial to demonstrate the effect of albumin administration in patients with hypoal- 
buminemia was the Saline versus Albumin Fluid Evaluation (SAFE) study [30], which included 7,000 critically ill patients. This study compared the effect of $4 \%$ albumin solution with that of normal saline, but there was no difference in 28-day mortality. However, in subgroup analysis, albumin therapy appeared to improve survival in patients with sepsis. A recent sepsis guideline suggests that albumin therapy can be considered in patients with severe sepsis who do not respond to crystalloid infusion [31]. Nevertheless, another recent study involving patients with sepsis admitted to the intensive care unit did not confirm the survival benefit of albumin infusion [32]. Although correction of hypoalbuminemia is a clinical issue, the present observational study could not prove the efficacy of albumin replacement in patients receiving CRRT

The present study has some limitations. In addition to the causal relationship issue, unidentified variables might have had a confounding effect on the relationship. As this was a single-center study, the characteristics of the study patients might have differed from those of patients in other cohorts or patients not undergoing CRRT. Finally, we did not consider whether the change in the albumin level or the replacement of albumin after CRRT was associated with mortality.

In conclusion, the present study addressed the predictive value of hypoalbuminemia with regard to shortterm and long-term mortality in patients starting CRRT. Although several factors might be associated with patient outcomes, serum albumin is beneficial in terms of its easy measurement in routine clinical practice. Accordingly, the serum albumin level should be monitored at CRRT initiation, and clinicians should be aware of the negative effects of hypoalbuminemia.

\section{Conflicts of interest}

All authors have no conflicts of interest to declare.

\section{Acknowledgments}

This work was supported by a grant from the Basic Science Research Program through the National Research Foundation of Korea (NRF) funded by the Ministry of Education (NRF-2017R1D1A1B03031642).

\section{Authors' contributions}

Jong Joo Moon participated in the data collection, analysis, and writing the manuscript. Yaerim Kim collected the data. Dong Ki Kim, Kwon Wook Joo, and Yon Su Kim interpreted the data and provided intellectual content of critical importance to the work. Seung Seok Han participated in the study design, interpretation of data, and revising the manuscript. All authors read and approved the final manuscript.

\section{References}

[1] Lameire NH, Bagga A, Cruz D, et al. Acute kidney injury: an increasing global concern. Lancet 2013;382:170-179.

[2] Mehta RL, Cerdá J, Burdmann EA, et al. International Society of Nephrology's 0by25 initiative for acute kidney injury (zero preventable deaths by 2025): a human rights case for nephrology. Lancet 2015;385:2616-2643.

[3] Cerdá J, Lameire N, Eggers P, et al. Epidemiology of acute kidney injury. Clin J Am Soc Nephrol 2008;3:881-886.

[4] Hoste EA, Bagshaw SM, Bellomo R, et al. Epidemiology of acute kidney injury in critically ill patients: the multinational AKI-EPI study. Intensive Care Med 2015;41:14111423.

[5] Srisawat N, Sileanu FE, Murugan R, et al.; Acute Kidney Injury-6 Study Group. Variation in risk and mortality of acute kidney injury in critically ill patients: a multicenter study. Am J Nephrol 2015;41:81-88.

[6] Uchino S, Kellum JA, Bellomo R, et al.; Beginning and Ending Supportive Therapy for the Kidney (BEST Kidney) Investigators. Acute renal failure in critically ill patients: a multinational, multicenter study. JAMA 2005;294:813-818.

[7] Bagshaw SM, Laupland KB, Doig CJ, et al. Prognosis for long-term survival and renal recovery in critically ill patients with severe acute renal failure: a population-based study. Crit Care 2005;9:R700-R709.

[8] Wald R, McArthur E, Adhikari NK, et al. Changing incidence and outcomes following dialysis-requiring acute kidney injury among critically ill adults: a population-based cohort study. Am J Kidney Dis 2015;65:870-877.

[9] Iwagami M, Yasunaga H, Noiri E, et al. Choice of renal replacement therapy modality in intensive care units: data from a Japanese Nationwide Administrative Claim Database. J Crit Care 2015;30:381-385.

[10] Quinlan GJ, Martin GS, Evans TW. Albumin: biochemical 
properties and therapeutic potential. Hepatology 2005;41: 1211-1219.

[11] Wilde B, Katsounas A. Immune dysfunction and albuminrelated immunity in liver cirrhosis. Mediators Inflamm 2019;2019:7537649.

[12] Roche M, Rondeau P, Singh NR, Tarnus E, Bourdon E. The antioxidant properties of serum albumin. FEBS Lett 2008; 582:1783-1787.

[13] Gabay C, Kushner I. Acute-phase proteins and other systemic responses to inflammation. N Engl J Med 1999;340: 448-454.

[14] Kaysen GA, Dubin JA, Müller HG, Rosales L, Levin NW, Mitch WE; HEMO Study Group NIDDK. Inflammation and reduced albumin synthesis associated with stable decline in serum albumin in hemodialysis patients. Kidney Int 2004;65:1408-1415.

[15] Herrmann FR, Safran C, Levkoff SE, Minaker KL. Serum albumin level on admission as a predictor of death, length of stay, and readmission. Arch Intern Med 1992;152:125-130.

[16] Lyons O, Whelan B, Bennett K, O’Riordan D, Silke B. Serum albumin as an outcome predictor in hospital emergency medical admissions. Eur J Intern Med 2010;21:17-20.

[17] Barchel D, Almoznino-Sarafian D, Shteinshnaider M, Tzur I, Cohen N, Gorelik O. Clinical characteristics and prognostic significance of serum albumin changes in an internal medicine ward. Eur J Intern Med 2013;24:772-778.

[18] Yu MY, Lee SW, Baek SH, et al. Hypoalbuminemia at admission predicts the development of acute kidney injury in hospitalized patients: a retrospective cohort study. PLoS One 2017;12:e0180750.

[19] Knaus WA, Draper EA, Wagner DP, Zimmerman JE. APACHE II: a severity of disease classification system. Crit Care Med 1985;13:818-829.

[20] Soeters PB, Wolfe RR, Shenkin A. Hypoalbuminemia: pathogenesis and clinical significance. JPEN J Parenter Enteral Nutr 2019;43:181-193.

[21] Knafl D, Vossen MG, Gerges C, et al. Hypoalbuminemia as predictor of recurrence of Clostridium difficile infection. Wien Klin Wochenschr 2019;131:68-74.

[22] Minatoguchi S, Nomura A, Imaizumi T, et al. Low serum albumin as a risk factor for infection-related in-hospital death among hemodialysis patients hospitalized on suspicion of infectious disease: a Japanese multicenter retrospective cohort study. Ren Replacement Ther 2018;4:30.

[23] Aldebeyan S, Nooh A, Aoude A, Weber MH, Harvey EJ. Hypoalbuminaemia-a marker of malnutrition and predictor of postoperative complications and mortality after hip fractures. Injury 2017;48:436-440.

[24] Sonoda A, Ohnishi S, Nakao S, et al. Factors affecting serum albumin in the perioperative period of colorectal surgery: a retrospective study. BMC Res Notes 2015;8:638.

[25] Cho YM, Choi IS, Bian RX, Kim JH, Han JY, Lee SG. Serum albumin at admission for prediction of functional outcome in ischaemic stroke patients. Neurol Sci 2008;29:445-449.

[26] Pavlakou P, Liakopoulos V, Eleftheriadis T, Mitsis M, Dounousi E. Oxidative stress and acute kidney injury in critical illness: pathophysiologic mechanisms-biomarkers-interventions, and future perspectives. Oxid Med Cell Longev 2017;2017:6193694.

[27] Daenen K, Andries A, Mekahli D, Van Schepdael A, Jouret F, Bammens B. Oxidative stress in chronic kidney disease. Pediatr Nephrol 2019;34:975-991.

[28] Wu CY, Hu HY, Huang N, Chou YC, Li CP, Chou YJ. Albumin levels and cause-specific mortality in community-dwelling older adults. Prev Med 2018;112:145-151.

[29] Suarez JI, Martin RH, Hohmann SF, et al. Human albumin use in adults in U.S. academic medical centers. Crit Care Med 2017;45:e16-e22.

[30] Finfer S, Bellomo R, Boyce N, French J, Myburgh J, Norton $\mathrm{R}$; SAFE Study Investigators. A comparison of albumin and saline for fluid resuscitation in the intensive care unit. $N$ Engl J Med 2004;350:2247-2256.

[31] Rhodes A, Evans LE, Alhazzani W, et al. Surviving sepsis campaign: international guidelines for management of sepsis and septic shock: 2016. Intensive Care Med 2017;43:304377.

[32] Caironi P, Tognoni G, Masson S, et al.; ALBIOS Study Investigators. Albumin replacement in patients with severe sepsis or septic shock. N Engl J Med 2014;370:1412-1421. 